\section{On the Interaction of a Premixed Flame with an Acoustic Disturbance}

\author{
Caroline Hood and Abdelkader Frendi ${ }^{1}$ \\ University of Alabama in Huntsville \\ Huntsville, AL, 35806
}

\begin{abstract}
The main objective of this research is to analyze the effect of acoustic disturbances on a premixed flame and determine their role in the onset of combustion instabilities. Computations for the one-dimensional, unsteady combustion of a lean premixed methaneair mixture are performed. An acoustic excitation is introduced in the chamber and interacts with the flame front. Our results indicate that as the amplitude of the acoustic excitation is increased, the flame front position fluctuates rapidly. This phenomenon is even more intense when the frequency of the acoustic disturbance matches the fundamental frequency of the chamber. Our results suggest that the interactions between the flame and the acoustic excitation may result in flame extinguishment. In addition various passive control devices are tested and we found that the Helmholtz resonator with rounded inlet corners is the most efficient.
\end{abstract}

\section{Introduction}

Combustion instabilities arise in many practical devices, such as rocket engines, aircraft engines, gas turbines,

\footnotetext{
${ }^{1}$ Corresponding author, Associate Professor, Senior Member of AIAA.
}

etc. Combustion instabilities are a concern in many engineering devices as they lead to malfunctioning of the device and a reduction in its efficiency. The sources of these instabilities are diverse and are dependent on fuetoxygen mixture, chamber geometry, injection mechanism, etc. It is believed that, combustion instabilities occur due to sustained pressure fluctuations of acoustic nature in a chamber where combustion takes place. These fluctuations alter the flame, which in turn drives sound through unsteady heat release.

It is well known that the resonance between a fluctuation in the heat release rate and an acoustic mode of the chamber is one of the main reasons for combustion instabilities. Prasad [1] investigated the effect of an external pressure perturbation on "premixed flames" using detailed chemistry. $\mathrm{He}$ solved the one-dimensional, time dependent, nonlinear conservation equations. His results showed that in response to the externally imposed pressure waves, the flame moves to a location where heat release and heat loss are in balance.

Peters and Ludford [2] concentrated on small variations of pressure and found that it was not affecting the flame significantly and hence concluded that substantially large variation in pressure (of the order of ambient pressure) might be needed to quench the flame. On the other hand, Ledder and Kapila [3] studied the response of a premixed laminar flame to pressure perturbations with emphasis on flame extinction. It was found that a very small variation in dynamic pressure has an influence on the burning rate, which could eventually lead to flame extinction. 
Due to the importance of combustion instabilities for different combustors, many studies have addressed the modeling and control of combustion instabilities in lean premixed mixtures. Paxson [4] modeled combustion instabilities in an LPP combustor using a sectored onedimensional code and studied the effect of an abrupt change of area in the combustor rig and how it expedites the phenomena of combustion instabilities using single-step kinetics. The author concludes that the position at which fuel is injected has a significant effect on acoustic instabilities.

Frendi [5] concentrated on the coupling between the unsteady heat release rate from combustion and the acoustic energy of the combustion chamber. Furthermore, he also studied the effect of adding a Helmholtz resonator and/or baffles to the system. $\mathrm{He}$ found that the use of a Helmholtz resonator and/or baffles shifts the frequency of the chamber away from the unstable mode.

In addition to the above studies, several other studies on different aspects of combustion instabilities have been carried out [6-14]. The basis for all the previous studies is due to Lord Rayleigh whose criteria states that the acoustic waves in the chamber will amplify if the acoustic pressure and heat release rate are "in phase".

In the present study, we concentrate on excitation of acoustic oscillations in combustion chambers, as it is an undesired phenomenon which results in inefficient burning and can lead to possible engine failure. However, accurate predictions of these types of acoustic instabilities are difficult to make as it primarily depends on the geometry of the combustor in question.
The work performed describes the interaction of an acoustic excitation with a premixed laminar flame in a closed, one-dimensional chamber. Depending on the excitation frequency and amplitude, this interaction leads to the growth of the pressure fluctuations in the system. At substantially high amplitude, the growth becomes nonlinear and results in flame extinction.

\section{Mathematical Formulation}

The Mathematical model consist of the Navier-Stokes equations combined with a one-step irreversible exothermic chemical reaction of the form

$$
v_{F} F+v_{O} O \rightarrow v_{P} P
$$

where " $F$ " is the fuel, " $O$ " the oxygen and "P" the products with $v_{i}$ the corresponding stoichiometric coefficients. All species are assumed to be in the gas phase.

The full set of conservation equations in one dimension are:

$$
\frac{\partial W}{\partial t}+\frac{\partial F}{\partial x}=B
$$

where,

$$
W=\left[\begin{array}{l}
\rho \\
\rho u \\
\rho e \\
\rho Y_{F} \\
\rho Y_{O}
\end{array}\right] \text {, }
$$




$$
F=\left[\begin{array}{l}
\rho u \\
\rho u^{2}+p-\frac{4}{3}\left(\mu \frac{\partial u}{\partial x}\right) \\
u(\rho e+p)-\left(\lambda \frac{\partial T}{\partial x}\right) \\
\rho u Y_{F}-\rho D_{F} \frac{\partial Y_{F}}{\partial x} \\
\rho u Y_{O}-\rho D_{O} \frac{\partial Y_{O}}{\partial x}
\end{array}\right],
$$

$$
B=\left[\begin{array}{l}
0 \\
0 \\
\dot{q}_{i g}^{m}+\dot{q}_{c o m b}^{m} \\
\dot{m}_{F}^{\prime \prime} \\
\dot{m}_{O}^{m}
\end{array}\right]
$$

In addition, the equation of state is given by

$$
p=\rho \frac{R}{\bar{M}} T
$$

with $\mathrm{p}$ being the pressure, $\rho$ the mixture density, $\mathrm{T}$ the temperature, $\mathrm{R}$ the universal gas constant and $\bar{M}$ the mixture molecular weight.

The source terms in the energy equations are given by

$$
\begin{aligned}
& \dot{q}_{c o m b}^{m}=h_{c}\left(\rho Y_{F}\right)^{m}\left(\rho Y_{O}\right)^{n} A \exp \left(\frac{-E_{a}}{R T}\right) \\
& \dot{q}_{i g}^{m}=\left[H(\tau)-H\left(\tau-\tau_{i g}\right)\right]^{*} \\
& \dot{E}_{i g}^{m} \exp \left[-g(P)\left(\frac{x-x_{0}}{r_{i g}}\right)^{P}\right]_{\rfloor}
\end{aligned}
$$

The source terms in the fuel and oxygen species conservation equations are

$$
\begin{aligned}
& \dot{m}_{F}^{m}=-v_{F} M_{F} \frac{\left(\rho Y_{F}\right)^{m}\left(\rho Y_{O}\right)^{n}}{\left(M_{F}\right)^{m}\left(M_{O}\right)^{n}} A_{r} \exp \left(\frac{-E_{a}}{R T}\right) \\
& \dot{m}_{O}^{m}=-v_{O} M_{O} \frac{\left(\rho Y_{F}\right)^{m}\left(\rho Y_{O}\right)^{n}}{\left(M_{F}\right)^{m}\left(M_{O}\right)^{n}} A_{r} \exp \left(\frac{-E_{a}}{R T}\right)
\end{aligned}
$$

The equations are solved in a nondimensional form using the following reference variables:

$$
\begin{aligned}
& l_{\text {ref }}=\left[\kappa_{\infty} t_{\text {ref }}\right]^{1 / 2}, \\
& t_{\text {ref }}=\left[\rho_{\infty}{ }^{2} Y_{O, r e f}{ }^{2} A \exp (-\beta)\right]^{-1}, \\
& u_{\text {ref }}=\left(l_{\text {ref }} / t_{\text {ref }}\right), \\
& e_{\text {ref }}=u_{\text {ref }}{ }^{2} \text { and } p_{\text {ref }}=\rho_{\infty} u_{\text {ref }}{ }^{2}
\end{aligned}
$$

\section{Method of Solution}

The set of PDE's are solved using an explicit finite difference scheme. The scheme, which is a generalization of the original MacCormack scheme obtained by Gottlieb and Turkel [15], is fourth order accurate in space and second order accurate in time. The numerical scheme applied to a one-dimensional equation of the form

$$
\frac{\partial U}{\partial t}=\frac{\partial F}{\partial x}
$$

consists of a predictor step given by 


$$
\begin{aligned}
& U_{i}^{* *}=U_{i}^{n}+ \\
& \left(\frac{\Delta t}{6 \Delta x}\right)\left(-7 F_{i}+8 F_{i+1}-F_{i+2}\right)
\end{aligned}
$$

followed by a corrector step of the form

$$
U_{i}^{n+1}=\frac{1}{2}\left(\begin{array}{l}
U_{i}^{* *}+U_{i}^{n}+ \\
\left(\frac{\Delta t}{6 \Delta x}\right)\left(7 F_{i}^{* *}-8 F_{i-1}{ }^{* *}+F_{i-2}^{* *}\right)
\end{array}\right)
$$

In the above equations, the subscript $i$ denotes the spatial grid point and the superscript $n$ denotes the time level. Superscript $(* *)$ corresponds to an intermediate time level (between $n$ and $(n+1))$. The fourth order accuracy is obtained by alternating the scheme given above with its symmetric variant. Furthermore, Jameson's [16] artificial dissipation terms were explicitly added to the finite difference scheme, which captured sudden jumps in pressure and other variables near shock waves and stagnation points. Adding these terms helped prevent the appearance of the well-known Gibbs phenomenon in regions containing severe pressure gradients. Both the inflow and outflow boundaries were rigid, $u=0$.

\section{Results and Discussions}

Consider a lean mixture of equivalence ratio $\phi=0.662$ in a closed chamber. Applying an external acoustic excitation at the inflow boundary of the form

$$
\tilde{u}=\varepsilon \sin (2 \pi f t+\Phi)
$$

where, $\tilde{u}$ is the non-dimensional velocity at the inflow boundary, $f$ the frequency, $\varepsilon$ the amplitude and $\Phi$ the phase.

The spark ignition parameters used for the present system are $r_{i g}=0.004 m, \quad \tau_{i g}=2.75 \mu \mathrm{s} \quad$ and $\dot{E}_{i g}^{m}=2.95 \cdot 10^{11} \mathrm{~J} / \mathrm{m}^{3} \mathrm{~s}$. The domain length is $0.2 \mathrm{~m}$. Gas properties for a lean mixture are different. We obtain the properties for the lean side from Coffee and Kotlar [17] and values corresponding to $\phi=0.662$ are given in Table 1. Two different cases are simulated based on changing frequency, a resonant case, $f=1940 \mathrm{~Hz}$, and an off-resonance case, $f=1730 \mathrm{~Hz}$.

\section{1) Resonant Case}

By changing the amplitude, $\varepsilon$, three cases were studied, with the use of the resonant frequency $f=1940 \mathrm{~Hz}$
(i) $\varepsilon=0$ - No Excitation
(ii) $\varepsilon=0.03$ - Low Excitation
(iii) $\varepsilon=0.3$ - High Excitation

Several observations have been made from the following figures. Figures 1, 2, 3 and show the temperature profiles at different times for $\varepsilon=0, \varepsilon=0.03$ and $\varepsilon=0.3$, respectively. In Figure 1 we observe that the temperature distribution is symmetrical and the flame fronts are traveling towards both boundaries at equal speed and the temperature of the flame front is settling down to the adiabatic temperature of the lean mixture, which is $T_{b}=2142.2 \mathrm{~K}$. Unlike the profile in Figure 1, the profiles in Figures 2 and 3 are different. The acoustic excitation results in increasing the speed of the positive propagating 
flame and slowing the negative propagating flame. Pressure time traces for different excitation amplitudes are shown in Figure 4, which shows the growth of the pressure oscillations with time and by increasing the amplitude.

\section{2) Off-Resonance Case}

By changing the amplitude, $\varepsilon$, three cases were simulated, with the use of off-resonance frequency of $f=1730 \mathrm{~Hz}$

(i) $\varepsilon=0$ - No Excitation

(ii) $\varepsilon=1.2$ - Low Excitation

(iii) $\varepsilon=2.4$ - High Excitation

Figures 5, 6 and 7 show the profiles of temperature at different excitation amplitudes and it can be observed that the effect on the flame front location as the amplitude is increased is very significant. Flame fronts are observed to move with unequal speeds towards both boundaries. Increasing the excitation amplitude, the inflow flame front is moving with a low speed.

Figures 8 compare the negative and positive propagating flame radius time histories for $\varepsilon=2.4$ with $\varepsilon=0$. By increasing the amplitude $(\varepsilon)$, the flame location fluctuates significantly which could lead to possible extinction.

Figure 9 shows the comparison of the time traces of the pressure at the center of the chamber at different times for the same amplitude $(\varepsilon=1.2)$ but different frequencies $(f=1730 \mathrm{~Hz}$ and $f=1940 \mathrm{~Hz}$ ). It is observed that pressure is growing much faster in the chamber when resonance frequency is imposed compare to the off-resonance frequency case.

\section{3) Passive Control}

Several passive control devices were tested numerically using a resonant acoustic disturbance alone, without combustion. The devices tested were, a baffle, a quaterwave and a Helmholtz resonator. In the later two cases, both smooth and sharp corners were considered. Figure 10 shows a comparison of the OASPL measured in the chamber the controlled and uncontrolled cases. It is clear from the figure that the Helmholtz resonator results in better control. Figure 11 shows the effect of rounding the various corners of the resonator. Our results show that rounding the inlet corners alone gives the best results. Figure 12 shows that the Helmholtz resonator is less effective at high sound pressure levels.

\section{Concluding Remarks}

Based on the results presented in this paper, the following conclusions are made:

1. The effect of the acoustic excitation is to displace the flame front. Depending upon the amplitude and frequency of the excitation, the flame front oscillations can be severe and may lead to flame extinc tion.

2. When the frequency of the acoustic excitation is at resonance with the fundamental frequency of the chamber, we found that the combustionacoustic interactions in the chamber resulted in rapid growth of the pressure oscillations. It can also be said that these nonlinear interaction between the heat 
release from the flame and the acoustic excitation may lead to a possible flame extinguishment.

3. The Helmholtz resonator with rounded inlet corners is the best passive control device tested.

\section{Acknowledgements}

Support for this research was provided by a grant from NASA Marshall Space Flight Center, Grant number NCC8-200, with Tom Nesman as the technical monitor.

\section{References}

[1] Prasad, K., "Interaction of Pressure Perturbations with Premixed Flames," Combustion and Flame, Vol. 97, 1994, pp.173-200.

[2] Peters, N. and Ludford, G.S.S., "The effect of pressure variations on premixed flames," Combustion Science

Technology, 34, 331.

[3] Ledder, G., and Kapila, A.K., "The Response of Premixed Flames to Pressure Perturbations," Combustion Science and Technology, 1991, Vol.76, pp. 21-44.

[4] Paxson, D., "A Sectored OneDimensional Model for Simulating Combustion Instabilities in Premix Combustors," Glenn Research Center, Cleveland, Ohio, 2000, AIAA-20000313.

[5] Frendi, A., "On the role of Acoustic Coupling on Combustion Instabilities," AIAA-2003-3181, Hilton Head, South Carolina, 2003.
[6] Wu, X., Wang, M., and Moin, P., "Combustion instability due to the nonlinear interaction between sound and flame," Center for Turbulence Research, Annual Research Briefs 2001.

[7] Matalon, M., and Matkowsky, B.J., "Flames as gas dynamics discontinuities," J. Fluid Mech. (1983), vol. 124 , pp. 239-259.

[8] Landau, L.D., "On the theory of slow combustion," $1944, \quad$ Acta Physicochimica URSS 19, 77.

[9] Umurhan, O.M., "Exploration of fundamental matters of acoustic instabilities in combustion chambers" Center for Turbulence Research, 1999.

[10] Lee, D.H., and Lieuwen, T.C., "Premixed Flame Kinematics in a Longitudinal Acoustic Field", AIAA-013851, Joint Propulsion Conference, 2001.

[11] Paschereit, C.O., Flohr, P., Gutmark, E., and Bockholts, M., "Numerically and Experimental Analysis of Acoustically Excited Combustion Instabilities in Gas Turbines" AIAA 2000-2076, Aeroacoustics Conference, June 2000.

[12] Culick, F.E.C., "A Note on Rayleigh's Criterion," Combustion and Science Technology, Vol. 56, 1987, pp. 159-166.

[13] Nguyen, Q. V., "Measurements of Equivalence Ratio Fluctuations in a Lean Premixed Prevaporized (LPP) Combustor and its correlation to Combustion Instability" National 
Aeronautics and Space Administration, Cleveland, $\mathrm{OH}$.

[14] Harper, J., Johnson, C., Neumeier, Y., Lieuwen, T., and Zinn, B.T., "Experimental Investigation of the Nonlinear Flame Response to Flow Disturbance in a Gas Turbine Combustor," AIAA-01-0486.

[15] Gottlieb, D., and Turkel, E., "Dissipative Two-Four Methods for Time-Dependent Problems," 1976, Mathematics of Computations, Vol. 30, No. 136, 703-723.

[16] Jameson, A., Schmidt, W., and Turkel, E., "Numerical Solutions of the Euler Equations by Finite Volume Methods Using Runga-Kutta TimeStepping Schemes," 1981, AIAA-811259.

[17] Coffee, T.P., Kotlar, A.J., and Miller, M.S., "The Overall Reaction Concept in Premixed, Laminar, Steady State Flames I. Stoichiometries," Combustion and Flame 54:155-169 (1983).

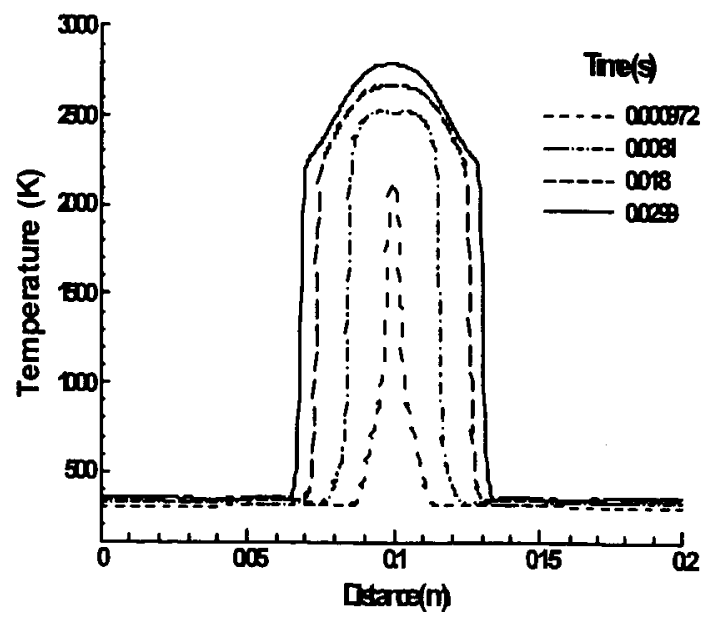

Figure 1: Temperature profiles in a "Closed Boundary System" as a function of time for a lean $(\phi=0.662)$ flame with no excitation $(\varepsilon=0), f=1940 \mathrm{~Hz}$

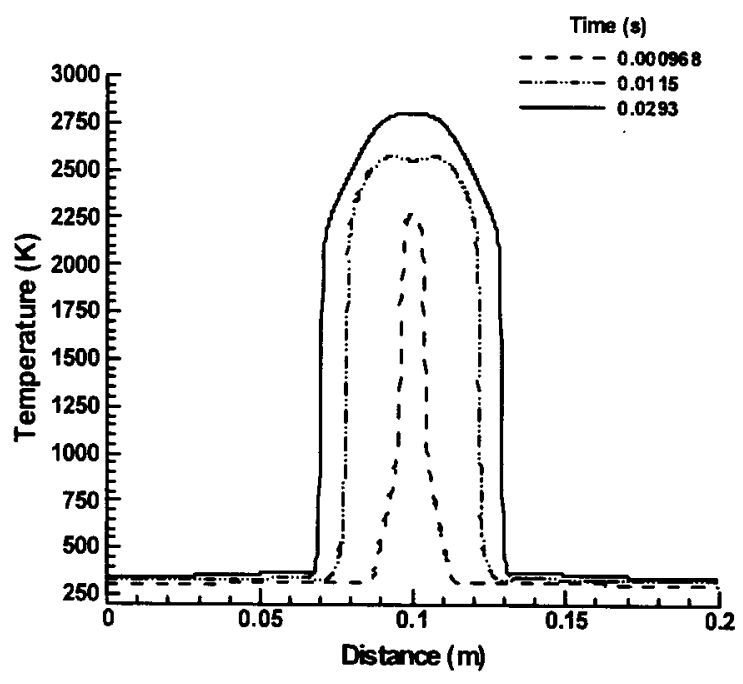

Figure 2: Temperature profiles in a "Closed Boundary System" as a function of time for a lean $(\phi=0.662)$ flame with low excitation $(\varepsilon=0.03), f=1940 \mathrm{~Hz}$

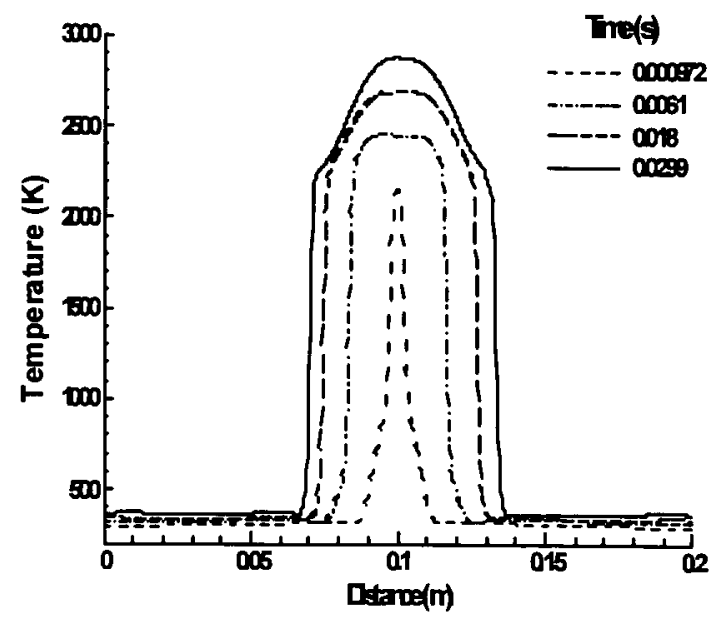

Figure 3: Temperature profiles in a "Closed Boundary System" as a function 
of time for a lean $(\phi=0.662)$ flame with low excitation ( $\varepsilon=0.3$ ), $f=1940 \mathrm{~Hz}$
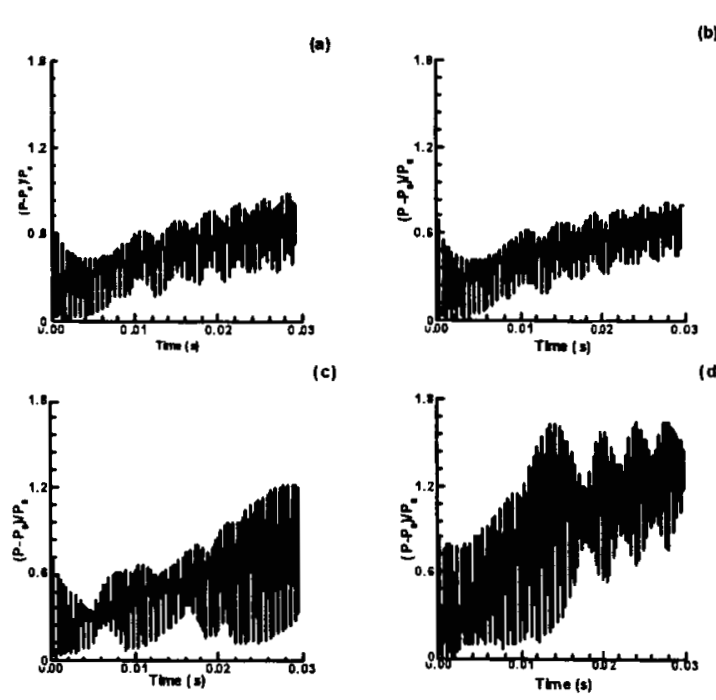

Figure 4: Time traces of pressure at the center of the chamber with different excitations for a lean mixture in a closed chamber (a) $\varepsilon=0$, (b) $\varepsilon=0.03$, (c) $\varepsilon=0.3$ and (d) $\varepsilon=1.2, f=1940 \mathrm{~Hz}$

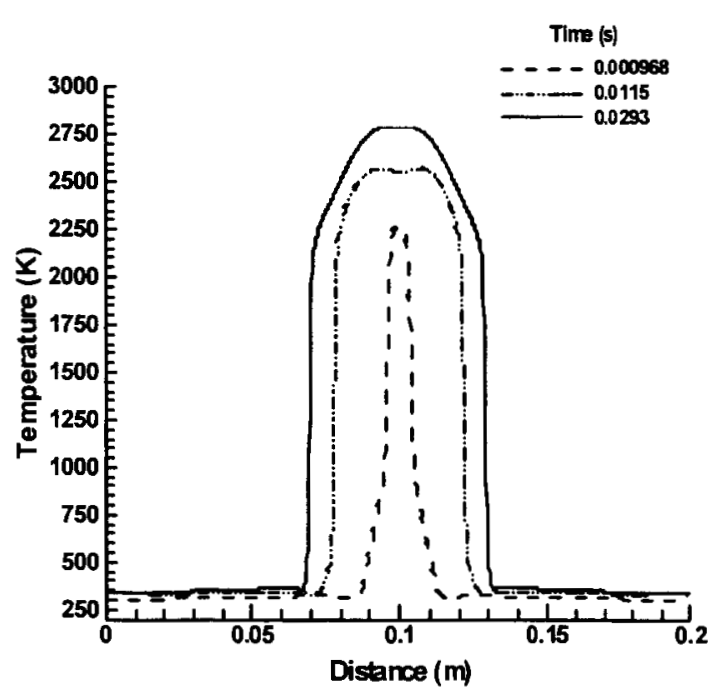

Figure 5: Temperature profiles in a "Closed Boundary System" as a function of time for

a lean $(\phi=0.662)$ mixture with no excitation $(\varepsilon=0), f=1730 \mathrm{~Hz}$.

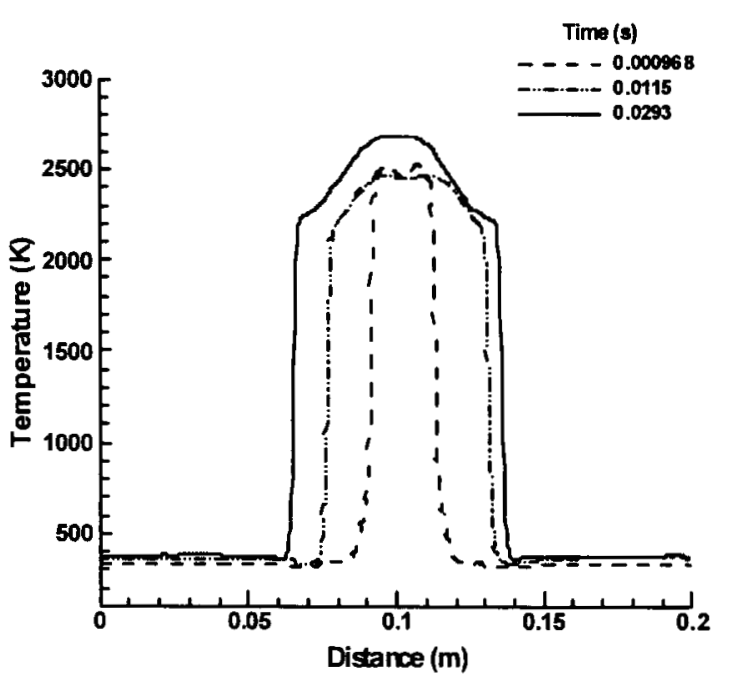

Figure 6: Temperature profiles in a "Closed Boundary System" as a function of time for a lean $(\phi=0.662)$ mixture with low excitation $(\varepsilon=1.2), f=1730 \mathrm{~Hz}$.

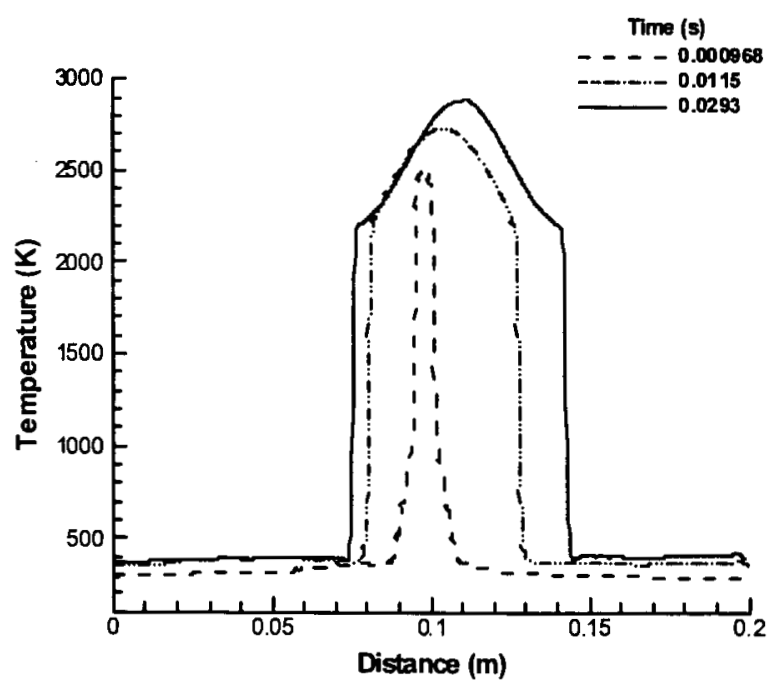

Figure 7: Temperature profiles in a "Closed Boundary System" as a function of time for a lean $(\phi=0.662)$ mixture with high excitation $(\varepsilon=2.4), f=1730 \mathrm{~Hz}$. 

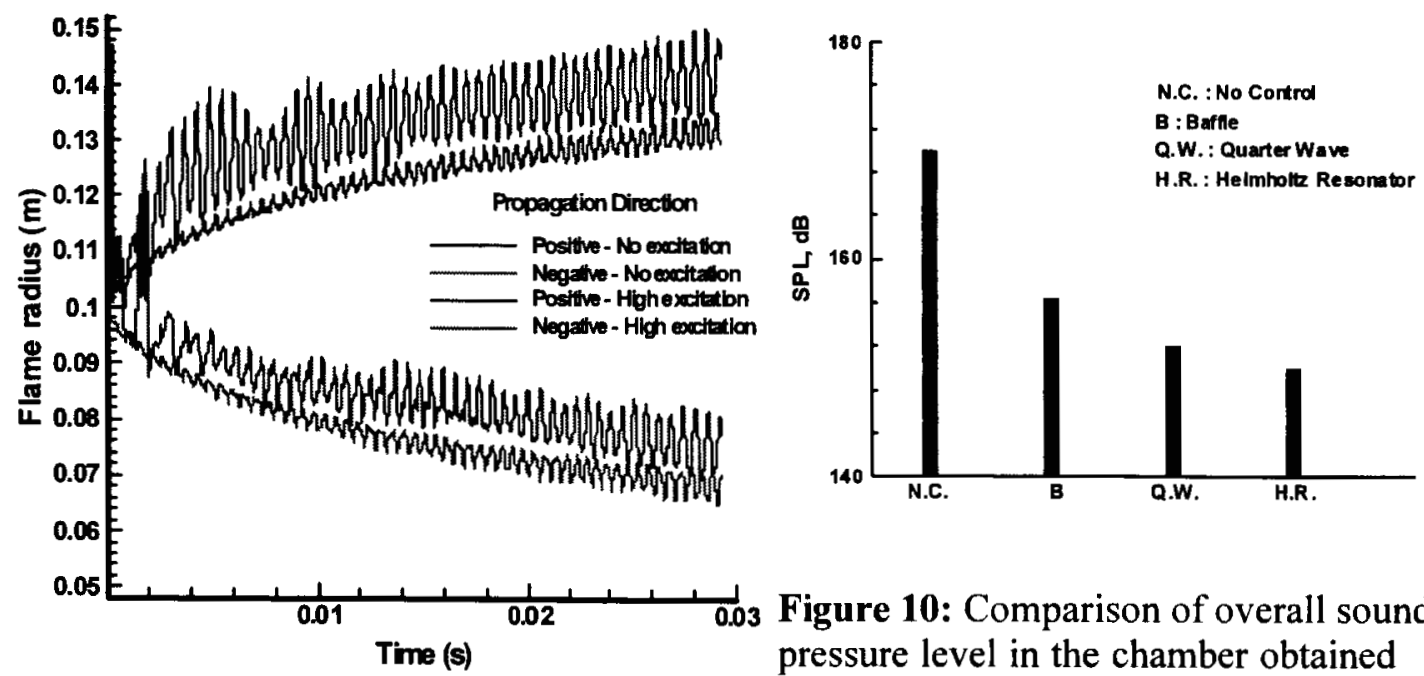

Figure 8: Flame trajectories as a function of time for $(\varepsilon=2.4) \quad$ and $(\varepsilon=0), \phi=0.662, f=1730 \mathrm{~Hz}$.

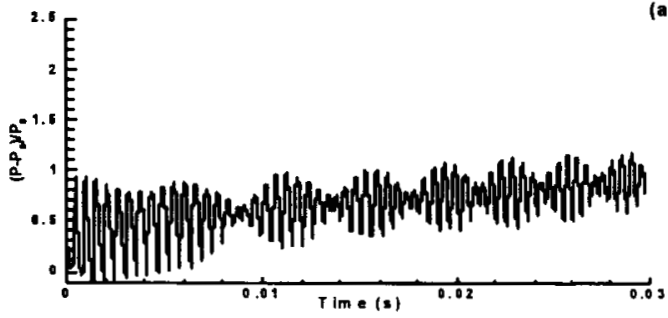

(a)
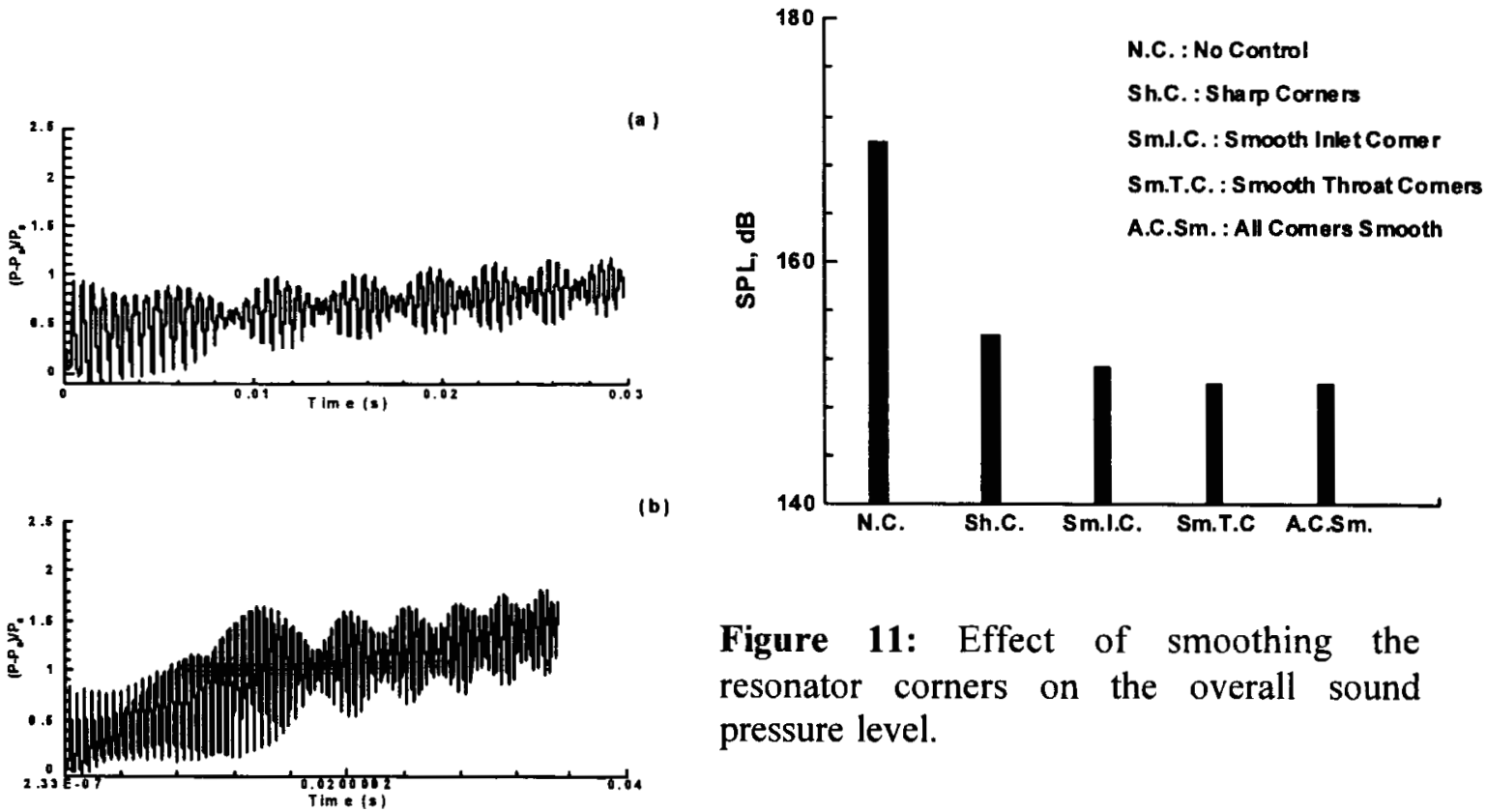

Figure 11: Effect of smoothing the resonator corners on the overall sound pressure level.

Figure 9: Time traces of pressure at the center of the chamber with different frequencies for a lean mixture in closed chamber (a) $f=1730 \mathrm{~Hz}$, (b) $f=1940 \mathrm{~Hz}$ for $\varepsilon=1.2, \phi=0.662$.

Figure 10: Comparison of overall sound pressure level in the chamber obtained with the various passive devices. 


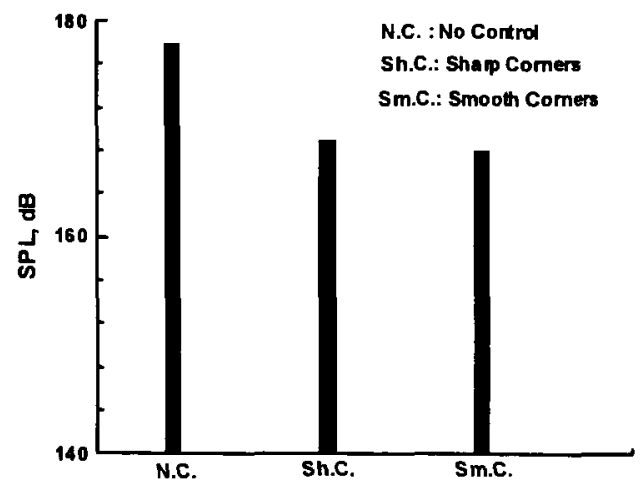

Figure 12: Effect of a Helmholtz resonator on the overall sound pressure level of a combustion chamber at high noise levels. 